\title{
Soziale Arbeit, Frauenbewegung, Internationales und Geschichte
}

\begin{abstract}
Ein Blick auf das Werk von Sabine Hering
Über Jahrzehnte hat Sabine Hering das Erscheinungsbild von Sozial Extra mitgeprägt. Mit über 60 Beiträgen zur Geschichte der Sozialen Arbeit und ihren herausragenden Persönlichkeiten, zu gesellschaftlichen Problemlagen und Selbstbehauptungsbestreben von Frauen, zu internationalen Perspektiven auf Soziale Arbeit und zuletzt insbesondere mit ihrem vehementen Plädoyer für ein Wohlfahrtsmuseum hat sie pointiert und kritisch den Fachdiskurs bereichert und herausgefordert.
\end{abstract}

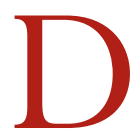
ie Beiträge dieses Schwerpunkts ermöglichen einen Blick zurück und bieten zugleich Ausblicke, wie an den von Sabine Hering angestoßenen Themen angeknüpft werden kann. Dass viele der Themen, die schon im letzten Jahrtausend auf der Tagesordnung der Sozialen Arbeit standen, auch heute noch nicht vom Tisch sind, zeigt der Reprint des Artikels „Von der Schande in die Sozialhilfe - Einblicke in eine 100jährige Geschichte alleinerziehender Frauen“ zur Situation von alleinerziehenden Frauen, den Sabine Hering 1988 in der Sozial Extra veröffentlicht hat.

Wie sie im Interview mit Barbara Lochner darlegt, gehört es zu ihren wesentlichen Anliegen, die Positionen von Frauen in der Gesellschaft wie auch in der Sozialen Arbeit zu stärken und zu fördern. Dafür fand und findet sie immer wie-

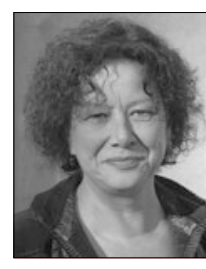

Leonie Wagner $* 1960$

Professorin für Pädagogik und Soziale Arbeit an der HAWK in Holzminden.

Leonie.Wagner@hawk-hhg.de

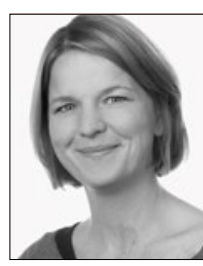

Barbara Lochner $* 1978$

Dr. phil., Dipl.-Sozialarbeiterin/-pädagogin (MA), wissenschaftliche Mitarbeiterin am Fachgebiet Erziehungswissenschaft mit dem Schwerpunkt Soziale Arbeit und außerschulische Bildung an der Universität Kassel barbara.lochner@uni-kassel.de der Mitstreiter_innen, wie die Beiträge von Leonie Wagner, Cornelia Wenzel und Elke Kruse belegen, die in Würdigung von Sabine Herings Werk, deren Themen und Formate aufgreifen. Leonie Wagner widmet sich in ihrem Beitrag dem Verhältnis der (neueren) Frauenbewegung und der Sozialen Arbeit. Sabine Herings Intention folgend, bedeutende Persönlichkeiten der Sozialen Arbeit vor dem Vergessen zu bewahren, erinnert Cornelia Wenzel an das Schaffen von Siddy Wronsky. Und Elke Kruse rekapituliert die Entwicklung internationaler Netzwerke in der Sozialen Arbeit. Keines dieser Themen steht einfach für sich. Das „Sozial-Extra-Gesamtwerk" von Sabine Hering vermittelt vielmehr ein kohärentes Bild zentraler Fragen in der Geschichte und Gegenwart der Sozialen Arbeit.

Im Sommer wird Sabine Hering 70 Jahre jung. Sie blickt auf ein bewegtes Leben zurück und hat noch immer sehr viel vor. Viel Erfolg dabei, we will miss you! Und nicht nur wir, wie die Grußbotschaften ehemaliger und derzeitiger Mitglieder des Sozial-Extra-Beirats zeigen. 